\title{
Le droit du travail confronté à la légalisation du cannabis
}

\section{Gilles Trudeau}

\section{Q OpenEdition}

1 Journals

\section{Édition électronique}

URL : https://journals.openedition.org/rdctss/2047

DOI : $10.4000 /$ rdctss. 2047

ISSN : 2262-9815

\section{Éditeur}

Centre de droit comparé du travail et de la sécurité sociale

\section{Édition imprimée}

Date de publication : 1 avril 2018

Pagination : 136-139

ISSN : 2117-4350

\section{Référence électronique}

Gilles Trudeau, «Le droit du travail confronté à la légalisation du cannabis », Revue de droit comparé du travail et de la sécurité sociale [En ligne], 1 | 2018, mis en ligne le 01 novembre 2021, consulté le 12 novembre 2021. URL : http://journals.openedition.org/rdctss/2047 ; DOI : https://doi.org/10.4000/ rdctss. 2047

\section{(c) (i) $\odot$}

Revue de droit comparé du travail et de la sécurité sociale est mise à disposition selon les termes de la Licence Creative Commons Attribution - Pas d'Utilisation Commerciale - Pas de Modification 4.0 International. 


\section{GILLES TRUDEAU}

UNIVERSITÉ DE MONTRÉAL

\section{LE DROIT DU TRAVAIL CONFRONTÉ À LA LÉGALISATION DU CANNABIS}

C'est le 13 avril 2017 qu'un projet de loi visant à légaliser le cannabis a été déposé devant le Parlement fédéral canadien'. Au moment d'écrire ces lignes, le projet de loi présentait toutes les chances d'être adopté et d'entrer en vigueur à l'été $2018^{2}$. II permet à tout adulte de 18 ans et plus de posséder du cannabis et d'y avoir accès en toute légalité. Le projet de loi fédéral confie aux provinces le soin de déterminer les règles applicables à la vente, la distribution et la consommation de cannabis sur leur territoire. Ainsi, au Québec, c'est à une agence gouvernementale, la Société québécoise du cannabis, que sera attribué le monopole de la vente du cannabis ${ }^{3}$. Le consommateur québécois pourra donc s'en procurer auprès de l'une des 200 succursales que la Société entend ouvrir d'ici deux ans, ou encore l'acheter en ligne directement à la Société.

Le projet de loi fédéral ne prévoit aucune mesure particulière relativement aux milieux de travail, ce que dénonce le mouvement patronal canadien. Les employeurs craignent que la légalisation du cannabis n'entraîne une hausse de sa consommation et que davantage de salariés ne se présentent intoxiqués au travail. On évoque un impact défavorable non seulement sur la productivité, mais surtout sur la sécurité au travail. Plusieurs réclament un cadre réglementaire établissant un seuil d'intoxication en milieu de travail, identifiant des appareils de détection certifiés et, surtout, permettant l'administration aléatoire de tests de dépistage aux salariés. Cette dernière mesure, perçue comme susceptible de décourager la consommation de cannabis, est particulièrement revendiquée par les employeurs des secteurs du transport.

Le droit canadien n'est pourtant pas insensible aux risques que présentent la consommation de drogues ou d'alcool et l'intoxication en milieu de travail. Il a, à cet égard, établi un certain équilibre entre l'exercice des prérogatives patronales et la protection des droits fondamentaux des salariés, particulièrement le droit à son intégrité physique et à la protection de sa vie privée ${ }^{4}$. Les employeurs canadiens prétendent que la légalisation du cannabis exige que cet équilibre soit modifié afin de leur conférer un pouvoir de contrôle accru.

1 Projet de loi C-45, Loi concernant le cannabis et modifiant la Loi réglementant certaines drogues et autres substances, le Code criminel et d'autres lois, $1^{\text {er }}$ session, 42e législature.

2 Le projet de loi a été adopté par la Chambre des Communes (Chambre basse) le 27 novembre

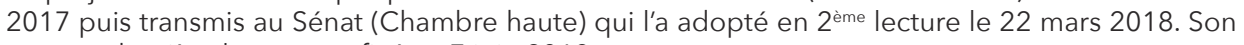
vote en dernière lecture est fixé au 7 juin 2018.

3 Projet de loi 157, Loi constituant la Société québécoise du cannabis, édictant la Loi encadrant le cannabis et modifiant diverses dispositions en matière de sécurité routière, $1^{\text {er }}$ session, $41 \mathrm{e}$ législature, déposé le 16 novembre 2017.

4 Voir sur ce point G. Trudeau, «Contrôle et prise en compte de la consommation de drogues et d'alcool par l'employeur au Québec », in Ph. Auvergnon (Dir.), Drogues illicites et activité salariée, Presses Universitaires de Bordeaux, Pessac, 2017, p. 129. 


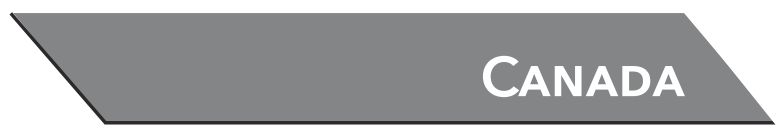

Une décision récente de la Cour suprême du Canada, rendue en juin 2017, présente un bon aperçu de l'état du droit canadien relativement à la consommation de drogues ou d'alcool au travail 5 . Elle met en lumière les principaux éléments de l'équilibre qui prévaut actuellement entre les prérogatives de l'employeur et les droits du salarié. L'employeur en cause, dont les activités dans la mine qu'il exploite sont dangereuses, adopte une politique relativement à la consommation de drogues, d'alcool et de médicaments chez ses salariés. Ces derniers sont tenus de déclarer tout problème de dépendance avant qu'un incident lié à la drogue ou à l'alcool ne survienne. Un traitement est offert à tous ceux qui se soumettent à une telle déclaration sans risque de sanction disciplinaire. Par contre, un salarié impliqué dans un incident et qui obtiendrait un résultat positif au test de dépistage auquel il serait obligatoirement soumis serait immédiatement licencié. C'est ce qui est arrivé au plaignant dans cette affaire, après qu'il eut été impliqué dans un accident au volant de son camion. Celui-ci consommait de la cocaïne lorsqu'il était en congé du travail, ce qu'il n'avait pas déclaré à l'employeur. Le test de dépistage qu'on lui administra après l'accident se révéla positif. Le plaignant fut licencié même s'il déclara à l'employeur, postérieurement à l'accident, croire souffrir d'une dépendance à la cocaïne. II contesta son licenciement qu'il prétendait discriminatoire parce que justifié par sa dépendance, un handicap au sens de la législation applicable. La Cour suprême ne retint pas cette prétention, confirmant plutôt que le licenciement avait été imposé pour contravention à la politique patronale.

La décision de la Cour suprême met d'abord en évidence la possibilité, pour l'employeur, de réglementer la consommation d'alcool et de drogues en milieu de travail. Ses pouvoirs de direction, que le contrat de travail conclu avec chaque salarié lui reconnaît, lui permettent d'adopter une directive à cet égard, et le justifie d'imposer une sanction disciplinaire au salarié qui y contreviendrait. La jurisprudence précise que pour qu'il en soit ainsi, le contenu de la directive doit être raisonnable, exprimé clairement et porté à la connaissance de chaque salarié. Une fois en vigueur, la directive doit être appliquée avec constance et uniformité, et le salarié doit être clairement avisé qu'y désobéir est susceptible d'entraîner l'imposition d'une sanction disciplinaire. Que la consommation du cannabis devienne légale n'empêchera pas l'employeur d'en défendre la consommation au travail pour autant. II est établi que consommer du cannabis diminue, à court terme, les capacités cognitives et la coordination motrice ${ }^{6}$. L'employeur a donc intérêt à prohiber l'usage de cannabis pendant les heures de travail et à empêcher que le salarié ne se présente au travail sous son influence. On peut à cet égard faire le parallèle avec l'alcool dont l'employeur peut défendre la consommation au travail, malgré la légalité d'en acheter et d'en consommer de façon générale.

Toutefois, la directive qui prohiberait toute consommation d'alcool ou de drogues à l'extérieur du travail risquerait d'être invalide, car l'employeur ne peut s'ingérer dans la vie personnelle du salarié à l'extérieur de son travail. Ce n'est que dans la mesure où la consommation d'une substance intoxicante par le salarié à l'extérieur du travail soit susceptible d'affecter sa prestation de travail que la directive patronale peut s'y intéresser. La consommation de drogues, dont le cannabis, et celle d'alcool doivent à cet égard être distinguées. Parce qu'il est difficile de détecter l'état d'un salarié qui se présenterait au travail sous l'effet d'une drogue, l'employeur pourrait en interdire la consommation à

5 Stewart c. Elk Valley Coal Corp., 2017 CSC 30.

6 Organisation mondiale de la santé, The health and social effects of non medical cannabis use, 2016, p. 40. 
l'extérieur du travail pour certaines catégories de salariés dont les fonctions le justifieraient comme, par exemple, les conducteurs d'autobus ou les pilotes d'avion. On peut croire que la légalisation du cannabis incitera davantage d'employeurs à agir en ce sens. L'interdiction patronale demeurera toutefois susceptible de contestation judiciaire, à l'occasion d'un licenciement pour y avoir contrevenu par exemple, et sa validité ne sera confirmée que si l'employeur démontre l'existence d'un risque tel, qu'il justifie l'atteinte au droit à la vie privée des salariés visés.

La décision de la Cour suprême susmentionnée évoque aussi la possibilité, pour l'employeur, de vérifier si un salarié est sous l'effet de la drogue ou de l'alcool en lui administrant un test de dépistage. Dans les circonstances propres à l'espèce, que l'employeur eut imposé un tel test ne fut pas contesté, et la Cour confirma la validité du licenciement en se fondant sur le résultat positif du test. La jurisprudence a toutefois limité strictement les circonstances autorisant l'employeur à imposer un test de dépistage à un salarié, cette mesure affectant considérablement le droit à la protection de la vie privée, généralement reconnu en droit canadien. L'imposition aléatoire de tests en l'absence d'un problème avéré de consommation de drogues ou d'alcool dans un milieu de travail est ainsi prohibée. La règle vaut même lorsque le travail est intrinsèquement dangereux. Dans ce cas, l'employeur ne pourra exiger d'un salarié qu'il se soumette à un test de dépistage que dans des circonstances précises, notamment si des motifs raisonnables permettent de croire que ses capacités sont affaiblies, s'il est impliqué directement dans un accident du travail ou un incident grave ou s'il revient au travail après avoir subi un traitement pour toxicomanie ${ }^{7}$. Ce sont ces conditions d'accès à l'imposition d'un test de dépistage que le mouvement patronal canadien juge trop restrictives dans le contexte de la légalisation de la consommation de cannabis.

Une difficulté supplémentaire s'ajoute quant à la légalité du recours aux tests de dépistage obligatoires en cas de drogues, dont le cannabis. Les tests actuellement disponibles permettent de savoir si le salarié a récemment consommé de la drogue mais ne permettent pas de déterminer quand le salarié a réellement consommé la drogue, ni s'il est encore sous son effet au moment de commencer à travailler ${ }^{8}$. Or, administrer un tel test de dépistage à un salarié peut s'avérer invasif et porter atteinte à ses droits fondamentaux, alors que son utilité est relative quant au risque que l'employeur cherche à éliminer. II en découle une légalité incertaine qui, dans plusieurs cas, doit être déterminée par le tribunal. De la même façon, les résultats du test ne sont que de peu de secours pour l'employeur qui cherche à établir que le salarié est actuellement intoxiqué et incapable d'exécuter adéquatement sa prestation de travail.

Intervient enfin, dans la décision rendue par la Cour suprême dans l'affaire Elk Valley, un dernier élément qui participe à l'équilibre que le droit canadien a établi entre les prérogatives patronales quant à la consommation de drogues et d'alcool dans le milieu de travail et le respect des droits des salariés. II s'agit de la protection particulière que la législation relative aux droits de la personne érige contre toute mesure patronale imposée

7 La Cour suprême du Canada rappelle cette règle dans Syndicat canadien des communications, de l'énergie et du papier, section locale 30 c. Pâtes \& Papier Irving, Ltée, 2013 CSC 34. Voir aussi: Teamsters Canada Rail Conference c. Canadian Pacific Railway Company, 2017 QCCA 479 (Cour d'appel du Québec).

8 Centre canadien d'hygiène et de sécurité au travail, Stratégie en milieu de travail: Risque de facultés affaiblies attribuable au cannabis, juin 2017, document nº DSS: CC273-2/17-3F, p.10. 
à un salarié à cause de son handicap. Les faits dans l'affaire Elk Valley l'illustrent bien. Le salarié fut licencié après avoir omis de déclarer sa dépendance à la cocaïne comme l'exigeait la directive patronale. La Cour jugea le licenciement non discriminatoire puisqu'à son avis, la dépendance du salarié n'en avait pas constitué un facteur. Les juges ont été convaincus par les preuves apportées que la dépendance à la cocaïne du salarié n'avait pas réduit sa capacité de respecter la directive de son employeur. Une conclusion contraire aurait signifié l'illégalité du licenciement.

Comme pour plusieurs autres drogues, l'usage du cannabis peut conduire à la dépendance", ce que le droit canadien assimile à un handicap. Le handicap constitue un motif illégal de discrimination sur lequel l'employeur ne peut fonder, directement ou indirectement, aucune mesure de représailles. Si la dépendance du salarié affecte sa capacité d'exécuter correctement sa prestation de travail, celui-ci faisant par exemple preuve d'un comportement inadéquat au travail ou d'un absentéisme excessif, l'employeur peut intervenir car il est en droit d'exiger une prestation de travail complète et satisfaisante. Il ne pourra toutefois le faire et, le cas échéant, licencié le salarié, qu'après s'être assuré qu'il lui est impossible de composer avec les limitations dues à son handicap sans en subir une contrainte excessive. L'employeur est en cela débiteur d'une obligation d'accommodement raisonnable.

L'ampleur de cette obligation varie selon les circonstances de chaque cas. Elle exige que l'employeur fasse de réels efforts pour accommoder le salarié, c'est-à-dire pour le maintenir en emploi malgré son handicap. Dans le cas d'un toxicomane, il peut s'agir de lui donner accès au programme d'aide aux travailleurs, dans la mesure où tel programme existe chez l'employeur, ou lui permettre de bénéficier d'un congé pour qu'il suive une cure de désintoxication. Les mesures d'accommodement peuvent aussi inclure un suivi administratif, ou prévoir que le salarié se soumette à un examen médical avant d'être autorisé à reprendre le travail. L'obligation d'accommoder le salarié est toutefois limitée, et cesse lorsque son exécution représenterait une contrainte excessive pour l'employeur. Là encore, l'existence d'une contrainte excessive ne peut s'apprécier qu'en tenant compte des circonstances de chaque cas. Généralement, le licenciement sera justifié lorsque, malgré les mesures d'accommodement prises, le salarié demeure incapable d'accomplir sa prestation de travail dans un avenir raisonnablement prévisible.

Les risques que la légalisation du cannabis fait craindre aux employeurs canadiens reposent sur deux hypothèses complémentaires: la banalisation de sa consommation d'une part et son augmentation de l'autre. Ces risques appréhendés justifient-ils de revoir l'équilibre que le droit canadien a établi entre l'exercice des prérogatives patronales relatives à la consommation de drogues et d'alcool en milieu de travail et la protection des droits fondamentaux des salariés? Les opinions sur ce sujet peuvent diverger mais, pour l'instant, il ne semble pas que les craintes patronales suscitent une réaction politique empressée.

9 Selon l'OMS, op.cit., supra note 6, p. 40, on a observé un risque de dépendance de $10 \%$ parmi les personnes ayant consommé du cannabis au cours de leur vie. Ce risque augmente à $16 \%$ lorsque la consommation survient pendant l'adolescence et à 33 \% parmi les consommateurs quotidiens. 